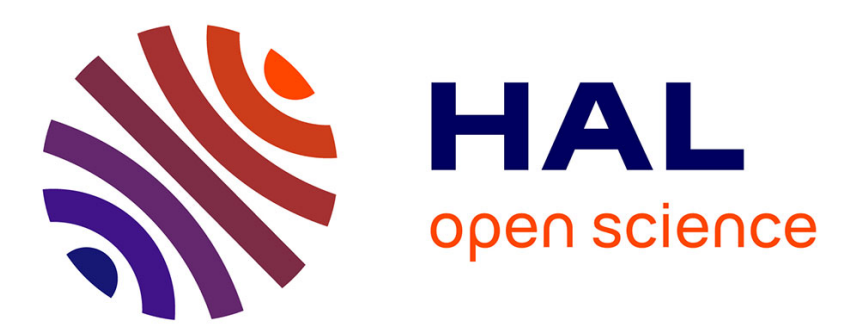

\title{
A convex programming bit allocation method for sparse sources
}

\author{
Mounir Kaaniche, Aurélia Fraysse, Beatrice Pesquet-Popescu, \\ Jean-Christophe Pesquet
}

\section{- To cite this version:}

Mounir Kaaniche, Aurélia Fraysse, Beatrice Pesquet-Popescu, Jean-Christophe Pesquet. A convex programming bit allocation method for sparse sources. Proceedings of the IEEE International Picture Coding Symposium (PCS), May 2012, Poland. pp.853-856. hal-00734456

\section{HAL Id: hal-00734456 https://hal.science/hal-00734456}

Submitted on 21 Sep 2012

HAL is a multi-disciplinary open access archive for the deposit and dissemination of scientific research documents, whether they are published or not. The documents may come from teaching and research institutions in France or abroad, or from public or private research centers.
L'archive ouverte pluridisciplinaire HAL, est destinée au dépôt et à la diffusion de documents scientifiques de niveau recherche, publiés ou non, émanant des établissements d'enseignement et de recherche français ou étrangers, des laboratoires publics ou privés. 


\title{
A convex programming bit allocation method for sparse sources
}

\author{
Mounir Kaaniche*, Aurélia Fraysse ${ }^{\dagger}$, Béatrice Pesquet-Popescu* and Jean-Christophe Pesquet ${ }^{\ddagger}$ \\ ${ }^{*}$ Télécom ParisTech, Signal and Image Processing Department, 46 rue Barrault, 75014 Paris, France. \\ E-mail: Email: $\{$ kaaniche,pesquet\} @ telecom-paristech.fr \\ † Univ Paris-Sud, Supélec, Laboratoire des Signaux et Systèmes, 3 rue Joliot-Curie, 91192 Gif-sur-Yvette cedex, France. \\ Email: fraysse@1ss.supelec.fr \\ ‡Université Paris-Est, Laboratoire d’Informatique Gaspard Monge, and CNRS UMR 8049, 77454 Marne-la-Vallée, France. \\ Email: jean-christophe.pesquet@univ-paris-est.fr
}

\begin{abstract}
The objective of this paper is to design an efficient bit allocation algorithm in the subband coding context based on an analytical approach. More precisely, we consider the uniform scalar quantization of subband coefficients modeled by a Generalized Gaussian distribution. This model appears to be particularly well-adapted for data having a sparse representation in the wavelet domain. Our main contribution is to reformulate the bit allocation problem as a convex programming one. For this purpose, we firstly define new convex approximations of the entropy and distortion functions. Then, we derive explicit expressions of the optimal quantization parameters. Finally, we illustrate the application of the proposed method to wavelet-based coding systems.
\end{abstract}

\section{INTRODUCTION}

In wavelet-based image coding systems, a natural ressource allocation question often arises: how a given bit budget can be efficiently distributed among the resulting subbands ? In other words, such a problem, referred to as the bit allocation problem, usually aims at finding the optimal quantization parameters satisfying some objective criterion. The standard criterion is based on Rate-Distortion (R-D) theory where the average distortion is minimized subject to a constraint on the available bitrate (or vice-versa). Therefore, it can be noticed that this constrained minimization problem requires the study of both the rate and the distortion functions. In the literature, two main approaches have been used to deal with this allocation problem. We distinguish the numerical- and analyticalbased methods. Numerical-based methods aim at empirically estimating the R-D functions and resort to some iterative techniques to find the optimal quantization parameters. Most of these methods are closely related to Lagrangian optimization techniques [13], [15]. In [13], a bit allocation method for completely arbitrary input signals (or blocks) and discrete quantizer sets was considered in the case of independent coding context. An extension of this work to the subband coding case has been proposed in [14]. Another extension to a dependent coding environment has been also considered in [10]. Moreover, it should be noticed that dynamic programming algorithms [9] and descent algorithms [11], [4] have also been proposed to select the optimal quantization parameters. It is important to note that most of these numerical methods are computationally intensive since a large number of R-D operating points have to be measured for each subband in order to obtain R-D curves which are often assumed to be both differentiable and convex [12]. In parallel, analytical approaches, which aim at finding closed-form expressions of the R-D functions by assuming various input distributions and quantizer characteristics, have been developed. For instance, the performance of optimum scalar quantizers subject to an entropy constraint was investigated through numerical methods [3] for different source probability densities (e.g uniform, Gaussian, Laplacian, Generalized Gaussian) at low resolution. In [1], a distortion measure based on differential entropy has been introduced for image coding and uniform scalar quantization. In this paper, we propose to design a novel bit allocation method in the subband coding context by adopting an analytical approach. More precisely, we present new approximations of the entropy and the distortion which allow us to reformulate the bit allocation problem by making use of convex analysis tools. Following this approach, we derive explicit expression of the optimal quantization parameters of the different subbands. The proposed optimization technique is suitable for transform-based image coders where Lagrangian optimization techniques are usually applied.

The remainder of this paper is organized as follows. In Sec. II, we define the probabilistic model of the considered wavelet subbands as well as the quantizer characteristics. New piecewise convex approximations of the entropy and distortion measures are given in Sec. III. The proposed bit allocation method is described in Sec. IV. Finally, in Sec. V, an application of the proposed method to transform coding is given and some conclusions are drawn in Sec. VI.

\section{SOURCE AND QUANTIZATION MODELS}

Let us assume that the source to be quantized is composed of $J$ subbands having $n_{j}$ coefficients $(j \in\{1, \ldots, J\})$ so that $n=\sum_{j=1}^{J} n_{j}$ is the total number of coefficients. The Generalized Gaussian (GG) model has been extensively employed for modelling the distribution of the wavelet coefficients [8]. The corresponding probability density function is given by:

$$
\forall \xi \in \mathbb{R}, \quad f_{j}(\xi)=\frac{\beta_{j} \omega_{j}^{1 / \beta_{j}}}{2 \Gamma\left(1 / \beta_{j}\right)} e^{-\omega_{j}|\xi|^{\beta_{j}}}
$$


where $\beta_{j}>0$ is the exponent parameter, $\omega_{j}>0$ is the scaling factor and $\Gamma$ is the gamma function.

These coefficients are then quantized by an uniform scalar quantizer with dead-zone, with a quantization step $q_{j}>0$, [6]. The output $\bar{X}_{j}$ of the quantizer is thus given by:

$$
\bar{X}_{j}=r_{0}=0, \quad \text { if }\left|X_{j}\right|<\frac{q_{j}}{2},
$$

and, for all $i \in \mathbb{Z}$ such that $i \neq 0$,

$$
\bar{X}_{j}=r_{i, j}, \quad \text { if }\left(|i|-\frac{1}{2}\right) q_{j} \leq\left|X_{j}\right|<\left(|i|+\frac{1}{2}\right) q_{j}
$$

where the reconstruction levels are given by

$$
\forall j \in\{1, \ldots, J\}, \quad \forall i \geq 1, \quad r_{i, j}=-r_{-i, j}=\left(i+\zeta_{j}\right) q_{j}
$$

and $\zeta_{j} \in[-1 / 2,1 / 2]$ is an "offset" parameter indicating the shift of the reconstruction level with respect to the middle of the quantization interval. Note that we will not consider any saturation effect. The most commonly used quantization rule corresponds to the case when $\zeta_{j}=0$. (i.e mid-interval reconstruction).

Since the objective of the paper is to focus on the bit allocation problem of the quantized coefficients, it is necessary now to study their rate and distortion functions. For this purpose, we approximate the bitrate by the zero-order entropy of the quantized coefficients [7]. Thus, the entropy of the associated uniformly quantized variable $\bar{X}_{j}$ is given by:

$$
H_{f_{j}}\left(q_{j}\right)=-\sum_{i=-\infty}^{\infty} \mathrm{P}\left(\bar{X}_{j}=r_{i, j}\right) \log _{2} \mathrm{P}\left(\bar{X}_{j}=r_{i, j}\right) .
$$

Furthermore, the distortion will be evaluated by the $p_{j}$-th order moment of the quantization error

$$
e_{f_{j}}\left(q_{j}\right)=\mathrm{E}\left[\left|X_{j}-\bar{X}_{j}\right|^{p_{j}}\right]
$$

where $p_{j} \geq 1$. We should note here that a close approximation of the entropy as well as asymptotic expressions of the distortion, at high and low resolutions, are given in [5]. However, these approximations have been derived for the case of $\log$-concave distributions (i.e for $\beta_{j} \in[1,2]$ ). Knowing that typical values of $\beta_{j}$ can be smaller than 1 in practice, we propose in this paper to consider new approximations which are also valid in the case when $0<\beta_{j}<1$. Once the entropy and the distortion functions have been introduced, let us now define new convex approximations of these functions.

\section{CONVEX APPROXIMATIONS OF THE ENTROPY AND THE DISTORTION}

Generally, analytical-based R-D algorithms use the standard Bennett formula to obtain a close approximation of the entropy [7], [5]. This formula allows us to express the entropy of the $j$-th subband as an affine function of $\mathbf{l}=\left(l_{1}, l_{2}, \ldots, l_{J}\right)$ :

$$
\widehat{H}_{f_{j}}\left(l_{j}\right)=-l_{j}+h_{\beta_{j}}\left(\omega_{j}\right)+o\left(l_{j} 2^{l_{j}}\right)
$$

where

- $\forall j \in\{1, \ldots, J\}, l_{j}=\log _{2}\left(q_{j}\right)$

- $h_{\beta_{j}}\left(\omega_{j}\right)$ is the differential entropy of the GG variable:

$h_{\beta_{j}}\left(\omega_{j}\right)=-\int_{-\infty}^{\infty} f(\xi) \ln f(\xi) d \xi=\log _{2}\left(\frac{2 \Gamma\left(1 / \beta_{j}\right)}{\beta_{j} \omega_{j}^{1 / \beta_{j}}}\right)+\frac{1}{\beta_{j}}$.
However, this approximation formula is only valid at high resolution (i.e when $q_{j}$ is small). In order to develop a bit allocation algorithm well-adapted for both high and low resolutions, we propose to replace the previous approximation of the entropy by another function of $\mathbf{l}=\left(l_{1}, l_{2}, \ldots, l_{J}\right)$, given by $\sum_{j=1}^{j} \frac{n_{j}}{n} g_{j}\left(l_{j}\right)$, where $g_{j}$ has a piecewise affine form defined for each $j \in\{1, \ldots, J\}$ by:

$\forall j \in\{1, \ldots, J\}, g_{j}\left(l_{j}\right)=a_{j}^{k} l_{j}+c_{j}^{k}$ if $l_{j}^{(h, k-1)} \leq l_{j} \leq l_{j}^{(h, k)}$

with $k \in\left\{1,2, \ldots, m^{(h)}\right\}$ and $m^{(h)}$ is a given parameter corresponding to the number of intervals (i.e. the number of segments defined to approximate the entropy). Thus, we suppose that $l_{j}^{(h, 0)} \leq l_{j}^{(h, 1)} \leq l_{j}^{(h, 2)} \leq \ldots<l_{j}^{\left(h, m^{(h)}\right)}$. Note that the superscript $(h)$ has been used to distinguish between the intervals of the approximation of the entropy and those of the approximation of the distortion. In order to have a decreasing function, the coefficients $\left(a_{j}^{k}\right)_{1 \leq k \leq m^{(h)}}$ should be negative. Furthermore, each coefficient $c_{j}^{k}$ is chosen such that $g_{j}$ remains a continuous function. Since the entropy must be a nonnegative function, we also impose that $g_{j}\left(l_{j}\right)=0, \forall l_{j} \geq l_{j}^{\left(h, m^{(h)}\right)}$. This condition allows us to deduce $l_{j}^{\left(h, m^{(h)}\right)}$ by finding the first point $l_{j}$ such that $g_{j}\left(l_{j}\right)=0$. Concerning the other points $\left(l_{j}^{(h, k)}\right)_{1 \leq k \leq m^{(h)}-1}$, they are chosen in a way that the resulting piecewise affine function is a good approximation of the entropy $H_{f_{j}}$. For example, for the first interval, it is well-known that a close approximation of the entropy at high bitrate is given by the Bennett formula, so leading to

$$
\forall j \in\{1, \ldots, J\}, \quad a_{j}^{1}=-1 \quad \text { and } \quad c_{j}^{1}=h_{\beta_{j}}\left(\omega_{j}\right)
$$

We thus obtain the piecewise affine function $g_{j}\left(l_{j}\right)$ on the first interval $\left[l_{j}^{(h, 0)}, l_{j}^{(h, 1)}\right]$. Then, we derive $\left(a_{j}^{2}, c_{j}^{2}\right)$ such that $g_{j}\left(l_{j}\right)$ on the interval $\left[l_{j}^{(h, 1)}, l_{j}^{(h, 2)}\right]$ is tangent to the entropy function at an arbitrary point $l_{j}>l_{j}^{(h, 1)}$. After that, the value of $l_{j}^{(h, 1)}$ can be selected by finding the configuration where the approximation error between the entropy $H_{f_{j}}$ and the function $g_{j}\left(l_{j}\right)$ on the interval $\left[l_{j}^{(h, 0)}, l_{j}^{(h, 2)}\right]$ is small. By following the same strategy, we deduce the remaining values $l_{j}^{(h, 2)}, l_{j}^{(h, 3)}, \ldots, l_{j}^{\left(h, m^{(h)}-1\right)}$. Figure 1 illustrates the approximations of the entropy for two intervals $\left(m^{(h)}=2\right)$ and four intervals $\left(m^{(h)}=4\right)$. As expected, increasing the number of intervals leads to a better approximation of the entropy. In a similar way, we use the following piecewise exponential approximation of the distortion (for an order moment $p_{j} \geq 1$ ):

$\forall j \in\{1, \ldots, J\}$,

$d_{j}\left(l_{j}\right)=\frac{\eta_{j}\left(p_{j}+1\right)}{\nu_{j}}\left(\alpha_{j}^{k} 2^{l_{j} \gamma_{j}^{k}}+\delta_{j}^{k}\right)$, if $l_{j}^{(d, k-1)}<l_{j} \leq l_{j}^{(d, k)}$

where

- $k \in\left\{1,2, \ldots, m^{(d)}\right\}$ and $m^{(d)}$ is a given parameter corresponding to the number of intervals used to approximate the distortion

- $\forall k \in\left\{1,2, \ldots, m^{(d)}\right\}, \alpha_{j}^{k}>0, \gamma_{j}^{k}>0, \delta_{j}^{k}$ and $\eta_{j}>0$ are some weighting factors 

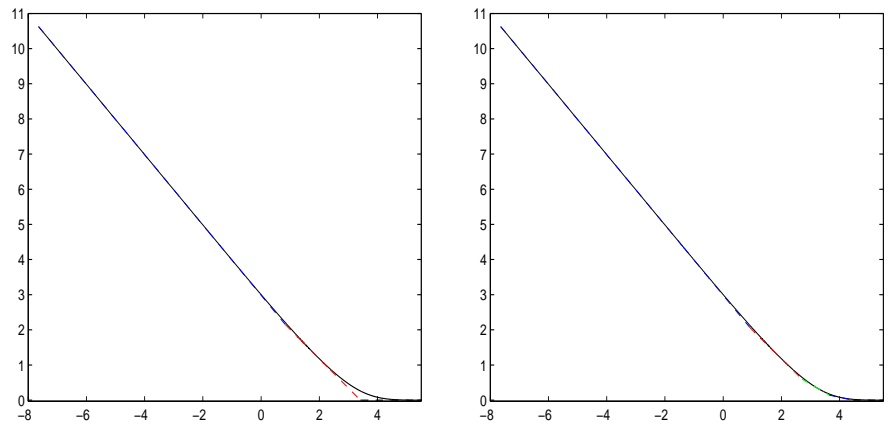

Fig. 1. Approximation $g_{j}$ (in color dashed line) of the entropy $H_{f_{j}}$ (in black line) of a uniformly quantized GG source versus $l_{j}: m^{(h)}=2$ (left side), $m^{(h)}=4$ (right side). The parameters of the GG source are $\beta_{j}=0.8$ and $\omega_{j}=1$.

- $\nu_{j}=\left(\frac{1}{2}+\zeta_{j}\right)^{p_{j}+1}+\left(\frac{1}{2}-\zeta_{j}\right)^{p_{j}+1}$.

Similarly to the selection procedure of $\left(l_{j}^{(h, k)}\right)_{1 \leq k \leq m}$, the values $\left(l_{j}^{(d, k)}\right)_{1 \leq k \leq m^{(d)}}$ are also chosen in a way that the resulting distortion function $d_{j}\left(l_{j}\right)$ is a good approximation of the distortion given by Eq. (6). In particular, by taking $\gamma_{j}^{1}=p_{j}, \eta_{j}=\alpha_{j}^{1}=\frac{\nu_{j}}{p_{j}+1}$, we obtain a distortion $d_{j}\left(l_{j}\right)=$ $\frac{\nu_{j}}{p_{j}+1} 2^{l_{j} p_{j}}$ on the first interval $\left[l_{j}^{(d, 0)}, l_{j}^{(d, 1)}\right]$ which corresponds to the classical asymptotic behavior of the distortion at high bitrate. Figure 2 shows the approximations of the distortion for 2 and 4 intervals. It can be observed that setting $m^{(d)}$ to 2 results in a less precise approximation of the distortion $e_{j}$ than $m^{(d)}=4$, especially at low bitrate.
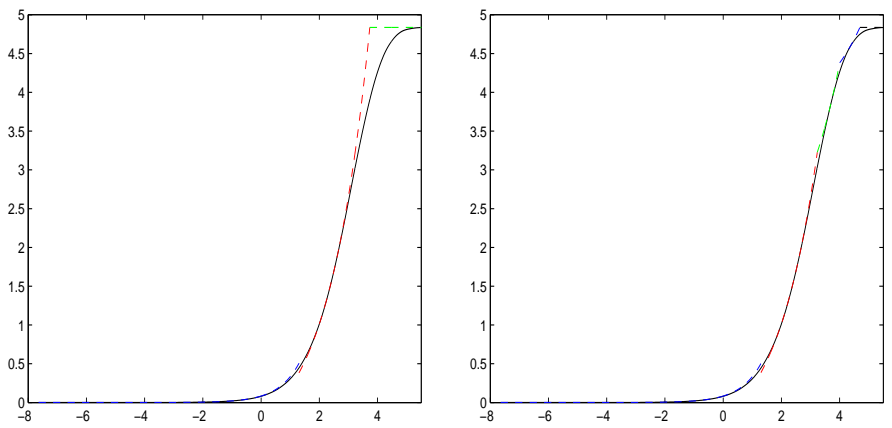

Fig. 2. Approximation $d_{j}$ (in color dashed line) of the distortion $e_{f_{j}}$ (in black line) of a uniformly quantized GG source versus $l_{j}: m^{(d)}=2$ (left side), $m^{(d)}=4$ (right side). The parameters of the GG source are $\beta_{j}=0.8$ and $\omega_{j}=1$.

\section{PROPOSED BIT ALLOCATION METHOD}

Once such approximations are obtained, we propose now to reformulate the bit allocation problem as an optimization problem as follows:

Problem 1: Find $\tilde{\mathrm{l}}$ minimizing the distortion function

$$
\forall \mathbf{l}=\left(l_{1}, \ldots, l_{J}\right) \in \mathbb{R}^{J}, \quad D(\mathbf{l})=\sum_{j=1}^{J} \frac{n_{j}}{n} d_{j}\left(l_{j}\right)
$$

over the set $C$ defined as

$$
C:=\left\{\mathbf{l}=\left(l_{1}, \ldots, l_{J}\right) \in \mathbb{R}^{J} \mid \sum_{j=1}^{J} \frac{n_{j}}{n} g_{j}\left(l_{j}\right) \leq R_{\max }\right\}
$$

A major difficulty that arises in solving this problem stems from the fact that the functions $g_{j}$ are not differentiable. In order to define the different domains where the optimization is performed, we sort the coefficients $\left(l_{j}^{(h, k)}\right)_{1 \leq k \leq m^{(h)}}$ and $\left(l_{j}^{(d, k)}\right)_{1 \leq k \leq m^{(d)}}$ in ascending order for each $j \in\{1, \ldots, J\}$. Thus, the sorted coefficients denoted by $\left(l_{j}^{1}, \ldots, l_{j}^{m}\right)$ are such that $l_{j}^{1} \leq l_{j}^{2} \leq \ldots \leq l_{j}^{m}$. Furthermore, let $l_{j}^{0}=$ $\min \left(\frac{n n_{j}^{-1} R_{\max }-c_{j}^{1}}{a_{j}^{1}}, l_{j}^{1}\right)$ for every $j \in\{1, \ldots, J\}$, so that the problem is equivalent to minimize the distortion over the domain $\left[l_{1}^{0}, l_{1}^{m}\right] \times \ldots \times\left[l_{J}^{0}, l_{J}^{m}\right]$. In order to overcome the problem of the non-differentiability of the functions $g_{j}$ at $\left(l_{j}^{k}\right)_{1 \leq k<m}$, we propose to subdivide the previous domain into subdomains of the form $\left[l_{1}^{b_{1}}, l_{1}^{b_{1}+1}\right] \times \ldots \times\left[l_{J}^{b_{J}}, l_{J}^{b_{J}+1}\right]$ where $\mathbf{b}=\left(b_{1}, \ldots, b_{J}\right) \in\{0, \ldots, m-1\}^{J}$. On each subdomain, the entropy and distortion functions are convex. Therefore, this subdivision technique leads to $m^{J}$ subdomains where a convex optimization problem must be solved.

Suppose that $\left[l_{1}^{b_{1}}, l_{1}^{b_{1}+1}\right] \times \ldots \times\left[l_{J}^{b_{J}}, l_{J}^{b_{J}+1}\right]$ corresponds to a given subdomain and let us denote by $\mathcal{P}_{\mathbf{b}}$ the convex minimization problem on this subdomain. For concision purposes, let us introduce the following notations for every $j \in\{1, \ldots, J\}$ :

$$
\begin{aligned}
& a_{j}=a_{j}^{b_{j}}, \gamma_{j}=\gamma_{j}^{b_{j}}, \alpha_{j}=\alpha_{j}^{b_{j}}, \delta_{j}=\delta_{j}^{b_{j}}, c_{j}=c_{j}^{b_{j}} \\
& N_{j}=-\frac{n_{j} a_{j}}{\gamma_{j}}, \underline{\lambda}_{j}=\kappa_{j} 2^{\gamma_{j} l_{j}^{b_{j}}}, \bar{\lambda}_{j}=\kappa_{j} 2^{\gamma_{j} l_{j}^{b_{j}+1}}
\end{aligned}
$$

where $\kappa_{j}=-\eta_{j} \alpha_{j} \gamma_{j} \ln 2 / a_{j}$.

Thus, the solution to Problem $\left(\mathcal{P}_{\mathbf{b}}\right)$ is given by:

\section{Proposition 1:}

(i) If $\sum_{j=1}^{J} \frac{n_{j}}{n} g_{j}\left(l_{j}^{b_{j+1}}\right)>R_{\max }$, then there is no solution.

(ii) If $\sum_{j=1}^{J} \frac{n_{j}}{n} g_{j}\left(l_{j}^{b_{j}}\right) \leq R_{\max }$, then the solution is $\widetilde{\mathbf{l}}=$ $\left(l_{1}^{b_{1}}, \ldots, l_{J}^{b_{J}}\right)$.

(iii) Otherwise, the solution is the vector $\widetilde{l}_{\mathbf{b}}$ defined by

$$
\forall j \in\{1, \ldots, J\}, \widetilde{l}_{j, \mathbf{b}}= \begin{cases}l_{j}^{b_{j}} & \text { if } j \in \mathbb{I} \\ \frac{1}{\gamma_{j}} \log _{2}\left(\frac{\tilde{\lambda}}{\kappa_{j}}\right) & \text { if } j \in \mathbb{J} \\ l_{j}^{b_{j}+1} & \text { if } j \in \mathbb{K}\end{cases}
$$

where

- $\widetilde{\lambda}^{N_{\mathbb{J}}}=$

$2^{-n R_{\max }+\sum_{j=1}^{J} n_{j} c_{j}-\sum_{j \in \mathbb{I}} N_{j} \gamma_{j} l_{j}^{b_{j}}-\sum_{j \in \mathbb{K}} N_{j} \gamma_{j} l_{j}^{b_{j}+1}} \prod_{j \in \mathbb{J}} \kappa_{j}^{N_{j}}$

- $N_{\mathbb{J}}=\sum_{j \in \mathbb{J}} N_{j}$.

- $\mathbb{I}=\left\{j \in\{1, \ldots, J\} \mid \Phi^{\prime}\left(\underline{\lambda}_{j}\right) \leq 0\right\}$

- $\mathbb{K}=\left\{j \in\{1, \ldots, J\} \mid \Phi^{\prime}\left(\bar{\lambda}_{j}\right)>0\right\}$

- $\mathbb{J}=\{1, \ldots, J\} \backslash(\mathbb{I} \cup \mathbb{K})$

- for every $j \in\{1, \ldots, J\}, \forall \lambda \in \mathbb{R}_{+}$

$$
\Phi(\lambda)=\lambda\left(\sum_{j=1}^{J} \frac{n_{j}}{n} c_{j}-R_{\max }\right)-\sum_{j=1}^{J} \varphi_{j}(\lambda)
$$




$$
\text { with } \varphi_{j}(\lambda)= \begin{cases}\frac{N_{j}}{n}\left(\gamma_{j} l_{j}^{b_{j}} \lambda-\frac{\lambda_{j}}{\ln 2}\right) & \text { if } \lambda \leq \underline{\lambda}_{j} \\ \frac{N_{j} \lambda}{n \ln 2}\left(\ln \left(\frac{\lambda}{\kappa_{j}}\right)-1\right) & \text { if } \underline{\lambda}_{j}<\lambda<\bar{\lambda}_{j} \\ \frac{N_{j}}{n}\left(\gamma_{j} l_{j}^{b_{j}+1} \lambda-\frac{\bar{\lambda}_{j}}{\ln 2}\right) & \text { if } \lambda \geq \bar{\lambda}_{j} .\end{cases}
$$

\section{APPLICATION TO TRANSFORM CODING}

In this part, we apply the proposed bit allocation method in the context of transform coding. For this purpose, we consider the example of "Elaine" image decomposed onto a Symlet othonormal wavelet basis of order 4 over 3 resolution levels (i.e. $J=10$ ). The parameters $\beta_{j}$ and $\omega_{j}$ of each subband are estimated using the maximum likelihood technique [2]. We illustrate in Fig. 3 (top side) the R-D curves where for each $j \in\{1, \ldots, J\}$, a quadratic distortion measure has been considered $\left(p_{j}=2\right)$. The PSNR curve plotted using the 'circle' symbol corresponds to the one predicted from Proposition 1 (i.e. resulting from quantizing the GG model). The PSNR curve plotted using the 'star' symbol is obtained by performing a uniform scalar quantization of the wavelet coefficient of the image with the derived optimal quantization steps. The comparison between the two distortion curves is performed by using different numbers of intervals to approximate both the entropy and the distortion. More precisely, we consider the cases $m^{(h)}=m^{(d)}=2, m^{(h)}=m^{(d)}=3$ and $m^{(h)}=m^{(d)}=4$. It can be noticed that the behavior of the proposed algorithm applied to a GG source model gets closer to the one applied on the image wavelet coefficients with increasing number of segments used for approximating the model. In addition, one can observe from Fig. 3 (top side) that the difference between the two distortion curves decreases as the interval number increases, which confirms our previous conclusions drawn from Figs. 1 and 2. Finally, compared with the state-of-the-art allocation method based on Lagrangian optimization technique [13] (green curve), our method achieves an improvement of about $0.3-0.7 \mathrm{~dB}$ as it can be seen in Fig. 3 (bottom side).

\section{CONCLUSION}

In this paper, we have proposed new piecewise convex approximations of the entropy and distortion measures in order to reformulate the bit allocation problem of sparse signals as a set of convex programming problems. The effectiveness of the proposed bit allocation method has been shown in a typical transform-based coding application.

\section{REFERENCES}

[1] T. André, M. Antonini, M. Barlaud, and R. Gray, "Entropy-based distortion measure and bit allocation for wavelet image compression," IEEE Trans. on Image Processing, vol. 16, no. 12, pp. 3058-3064, 2007.

[2] M. N. Do and M. Vetterli, "Wavelet-based texture retrieval using generalized Gaussian density and Kullback-Leibler distance," IEEE Transactions on Image Processing, vol. 11, no. 2, pp. 146-158, 2002.

[3] N. Farvardin and J. W. Modestino, "Optimum quantizer performance for a class of non-Gaussian memoryless sources," IEEE Transactions on Information Theory, vol. 30, no. 3, pp. 485-497, 1984.

[4] K. Ferguson and N. Allinson, "Modified steepest-descent for bit allocation in strongly dependent video coding," IEEE Transactions on Circuits and Systems for Video Technology, vol. 19, no. 7, pp. 1057-1062, 2009.

[5] A. Fraysse, B. Pesquet Popescu, and J.-C. Pesquet, "On the uniform quantization of a class of sparse sources," IEEE Trans. on Information Theory, vol. 55, no. 7, pp. 3243-3263, 2009.
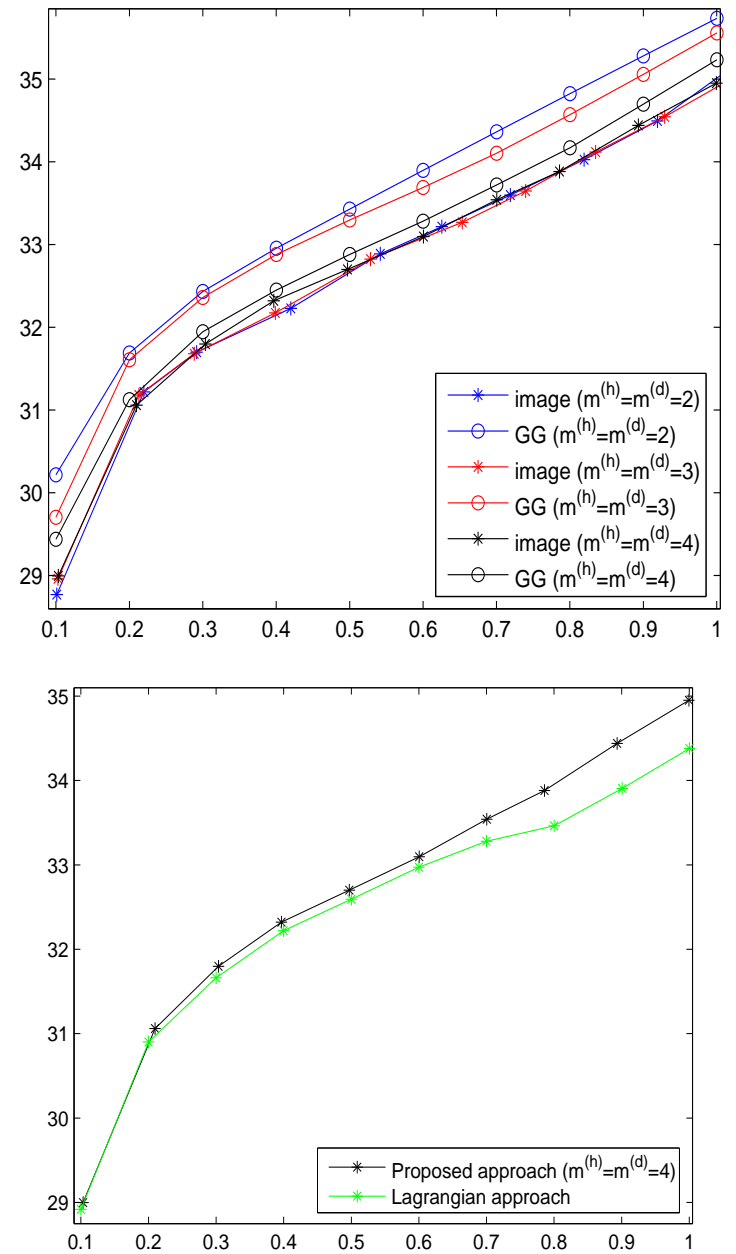

Fig. 3. PSNR (in $\mathrm{dB}$ ) versus the entropy (in bpp) following an uniform scalar quantization of the "Elaine" image: influence of the number of intervals (top), performance of the proposed approach vs the Lagrangian one (bottom).

[6] A. Gersho and R. Gray, Vector Quantization and Signal Compression. Boston, MA: Kluwer, 1992.

[7] H. Gish and J. Pierce, "Asymptotically efficient quantizing," IEEE Transactions on Information Theory, vol. 14, no. 5, pp. 676-683, 1968.

[8] S. Mallat, "A theory for multiresolution signal decomposition: The wavelet representation," IEEE Transactions on Pattern Analysis and Machine Intelligence, vol. 11, pp. 674-693, 1989.

[9] A. Ortega and K. Ramchandran, "Rate-distortion methods for image and video compression," IEEE Sig. Proc. Mag., vol. 15, pp. 23-50, 1998.

[10] K. Ramchandran, A. Ortega, and M. Vetterli, "Bit allocation for dependent quantization with applications to multiresolution and MPEG video coders," IEEE Trans. on Image Proc., vol. 3, no. 5, pp. 533-545, 1994.

[11] E. A. Riskin, "Optimal bit allocation via the generalized BFOS algorithm," IEEE Trans. on Inf. Theory, vol. 37, no. 2, pp. 400-402, 1991.

[12] Y. Sermadevi and S. S. Hemami, "Convexity results for a predictive video coder," in Asilomar Conference on Signal, Systems and computers, vol. 2, CA, November 2004, pp. 1713-1717.

[13] Y. Shoham and A. Gersho, "Efficient codebook allocation for an arbitrary set of vector quantizers," International Conference on Acoustics, Speech, and Signal Processing, vol. 10, pp. 1696-1699, 1985.

[14] P. Westerink, J. Biemond, and D. Boekee, "An optimal bit allocation algorithm for sub-band coding," International Conference on Acoustics, Speech, and Signal Processing, pp. 757-760 vol.2, 1988.

[15] S.-W. Wu and A. Gersho, "Rate-constrained optimal block-adaptive coding for digital tape recording of HDTV," IEEE Trans. on Circuits and Systems for Video Technology, vol. 1, no. 1, pp. 100-112, 1991. 